\title{
A Fusion Model for Securities Analysts' Stock Rating Information Based on the Evidential Reasoning Algorithm under Two-dimensional Progressive Recognition Framework
}

\author{
Weidong Zhu ${ }^{1}$, Yiling $\mathrm{Wang}^{2}$, Yong $\mathrm{Wu}^{3}$ and Yibo $\mathrm{Sun}^{4}$ \\ ${ }^{1}$ School of Economics, Hefei University of Technology, Hefei, Anhui, 230009, \\ China \\ Key Laboratory of Process Optimization and Intelligent Decision-making, \\ Ministry of Education, Hefei, Anhui, 230009, China \\ ${ }^{2}$ School of Management, Hefei University of Technology, Hefei, Anhui, 230009, \\ China; \\ ${ }^{3}$ School of Management, Hefei University of Technology, Hefei, Anhui, 230009, \\ China \\ Key Laboratory of Process Optimization and Intelligent Decision-making, \\ Ministry of Education, Hefei, Anhui, 230009, China \\ ${ }^{4}$ School of Management, Hefei University of Technology, Hefei, Anhui, 230009, \\ ${ }^{1}$ zhuwd@hfut.edu.cn, ${ }^{2}$ wangyiling@mail.hfut.edu.cn, \\ 3wuyong@hfut.edu.cn, yibo2237@126.com
}

\begin{abstract}
Securities analysts' forecast information can effectively reduce the uncertainty of information in securities markets, and can also promote effective allocation in capital market. The personality difference of securities analysts will lead to different analysis results. In order to improve the utilization of analysts' forecast information, evidential reasoning algorithm under two-dimensional progressive framework and grouping method for combining evidence were used in this paper to fuse securities analysts' stock rating information. Based on the forecast earnings information and stock rating information of analysts, we constructed a two-dimensional progressive framework, and then fused stock rating information of multiple analysts into one piece of evidence information. Finally, we empirically verified the model in this paper by using Chinese analysts' forecast information. The analysis on the fusion results have shown that: compared to traditional statistic model, the accuracy, certainty and the discrimination of the fusion results in our model have been improved.
\end{abstract}

Keywords: Securities Analyst; Stock Rating; Two-dimensional Progressive Recognition Framework; Information Fusion; Evidential Reasoning

\section{Introduction}

Securities analyst will analyze and forecast the value or changing trends of securities market or a listed firm, based on their professional skill and advantages in obtaining information from unique channels. Then he would make a research report and publish it to investors or public, and provide some valuable investment advice [1]. There are two common research reports, one is the earnings forecast information of listed firms, and the other is the stock rating information. Securities analysts' forecast data contains a generous amount of information, which can effectively decrease the informational asymmetry in the market [2]. 
For one stock, there may be several analysts to study and forecast. Analyst will provide the final rating information (stock rating) [3] of the stock after the consideration of all his resources, such as insider information, professional knowledge, etc. Investors, often individuals (private investor) would pay much attention to analysts' stock rating information. While analysts have difference in education and working experiences, they will have different views on the same stock, even contradictory. Research on analysts has focused on the forecast accuracy and its effect factors of analysts [4-6], the independence of analysts [7] and the forecast behavior [8]. Much less attention has been paid to the fusion of analysts' forecast information. Fusing securities analysts' forecast information with conflict, not only will make a contribution to investors, but also contributes to the effective allocation in capital market.

Traditional analysts' information analysis method is based on statistical methodology [9]. The analytic process is as follows: first, summarizes all the forecast information of analysts of one stock, and figures out each analyst's stock rating; second, analyzes each rating based on simple statistical formula; generally the result is represented by the ratio between the number of analysts, who gives the rating, and the total number of analysts.

In the fusion of analysts' stock rating forecast information based on statistical methodology, the importance of all the analysts has been given equal. But the professional knowledge and practical experience are different in analysts [10], so the forecast accuracy of analysts will be different. In recent years, some institutions have made a comparison to analysts, such as the "star analysts" of New Fortune Magazine.

We considered that there are two deficiencies in the information fusion based on statistical methodology:

(1) Analysts' individual differences have not been considered, thus the reliability and accuracy of the fusion results will be decreased.

(2) Fusion based on statistical methodology is not a fusion of analysts' forecast information, but a statistical number of analysts of each stock rating. From the perspective of systems theory, traditional fusion treated all the forecast information as an information source, which has weakened analysts' individual effect. Each expert must be treated as an information source in the fusion.

Evidence theory [11] has unique advantages in information fusion, and it has been widely used in areas, such as information fusion [12], fault diagnosis [13], multiple attribute group decision making [14], etc. Yang Shanlin, Zhu Weidong, et. al., [15] has applied traditional evidence theory to fuse analysts' forecast information, and evidence correction factor was obtained through the numerical relationship of analysts' forecast information by using the adaptive learning of neural network. In order to overcome the deficiencies in the fusion based on statistical methodology and improve the accuracy of fusion results, on the basis of paper [15] this paper took the characteristic information of analysts into account, evidential reasoning algorithm under two-dimensional progressive framework and grouping method for combining evidence were applied to fuse multiple securities analysts' forecast information with conflicts in this paper.

\section{Theoretical Fusion Model for Analysts' Information under Two-dimensional Progressive Recognition Framework}

\subsection{Grouping Method for Combining Evidence under Two-dimensional Progressive Recognition Framework}

Considering the difference of characteristic of information sources, we added a second dimensional evidence information framework $\Psi$ on the traditional evidence framework $\Theta$ to describe the attributive characteristic of evidence resources. Thus a two-dimensional 
progressive evidence recognition framework [16] $\{\Psi \rightarrow \Theta\}$ is constructed, which can be used in information fusion and has adequately considered the difference of characteristic of the evidence.

Learn from the grouping method for combining evidence based on the characteristic of evidence resources under two-dimensional progressive recognition framework, which was proposed in our early paper [17]:

Step 1, analyze the data and problems, and then construct the two-dimensional progressive recognition framework.

Step 2, group all evidence resources base on the second dimensional information framework $\Psi$, and analysts with similar characteristic will be grouped together: $\left(E_{G 1}, E_{G 2}, \ldots, E_{G i}\right)=\left\{\left(E_{1}, E_{2}, \ldots, E_{n}\right) \mid \Psi\right\}$, where $E_{G i}$ represents the evidence of group i.

Step 3, compute the weights of each evidence in the same group based on the diversity of the evidences. By taking a weighted average of the basic probability in the group, fuse the evidence information of each group, and the fusion result of each group is $B P A_{G i}$ :

$B P A_{G i}=\sum_{B P A_{j} \leq E_{G i}} \omega_{j} B P A_{j}$

Step 4, build a mapping function, which the characteristic of the analysts in each group can be mapped to evidence correction factor $\alpha$, and $\alpha \in[0,1]$, correct BPA by $\alpha$, and then fuse the information using Dempster' combination rule [12].

$m^{\alpha}(A)=\left\{\begin{array}{cc}\alpha * m(A) & A \in 2^{\Theta}, A \neq \varnothing \\ (1-\alpha)+\alpha * m(A) & A=\Theta\end{array}\right.$

$m(A)= \begin{cases}0, & A=\phi \\ \frac{\sum_{A_{i} \wedge B_{j}=A} m_{1}^{\alpha}\left(A_{i}\right) m_{2}^{\alpha}\left(B_{j}\right)}{1-\sum_{A_{i} \cap B_{j}=\phi} m_{1}^{\alpha}\left(A_{i}\right) m_{2}^{\alpha}\left(B_{j}\right)}, & A \neq \phi\end{cases}$

\subsection{Fusion Model of Analysts' Information based on Grouping Method for Combining Evidence}

2.2.1. Construction of the two-dimensional progressive framework: Stock rating is analysts' final opinion on a stock, it is also the information resource of the fusion model in this paper. The purpose of our model is to fuse the stock rating information of analysts. Stock rating of analysts was treated as the element of traditional evidence framework (the first dimensional framework). In order to have an unified standard in the fusion, the analysts' forecast data was mapped to five levels, they are buy, outperform, neutral, reduction, sell. Thus, there are five elements in the traditional evidence framework, they are buy $\left(\theta_{1}\right)$, outperform $\left(\theta_{2}\right)$, neutral $\left(\theta_{3}\right)$, reduction $\left(\theta_{4}\right)$, sell $\left(\theta_{5}\right)$. Hence, the first dimensional evidence framework is $\Theta=\left\{\theta_{1}, \theta_{2}, \theta_{3}, \theta_{4}, \theta_{5}\right\}$.

Analysts' earnings forecast information mainly includes the forecast information of financial performance of listed firms, such as earnings per share (EPS), price earnings ratio, net profit, major business income, earnings before interest and tax, etc. The earnings forecast information can represent the individual characteristic of analysts in some degree, such as analyst's ability (obtaining and analyzing information), evaluation standard and optimistic tendency, etc. Hence, the second dimensional framework is based on the earnings forecast information of listed firms, and the purpose of the second dimensional evidence framework is to measure the errors of analyst's earnings forecast. The correction 
factor can be obtained by constructing an evidence correction function based on the forecast error.

In most studies, scholars choose the forecast error of EPS to measure the forecast error of analysts (the difference between the forecast EPS and actual EPS). To simplify the model, and increase its computational efficiency, the second dimensional evidence framework is a singleton set only includes EPS $(\varphi)$. The EPS information includes both analysts' forecast EPS information $\left(\varphi_{1}\right)$ and the actual EPS information $\left(\varphi_{2}\right)$. Thus, the second dimensional evidence framework is: $\Psi=\left\{\varphi / \varphi_{1}, \varphi_{2}\right\}$.

In conclusion, the two-dimensional progressive framework in information fusion model for stock rating of securities analysts is: $(\Psi ; \Theta)=\left(\left\{\varphi / \varphi_{1}, \varphi_{2}\right\} ;\left\{\theta_{1}, \theta_{2}, \theta_{3}, \theta_{4}, \theta_{5}\right\}\right)$.

2.2.2. Evidence Grouping under the Second Dimensional Framework: The second dimensional framework is the relevant information about EPS: forecast EPS information, $\operatorname{FEPS}\left(\varphi_{1}\right)$, and the actual EPS information, $\operatorname{AEPS}\left(\varphi_{2}\right)$. We grouped the experts though the EPS information. Cao Sheng \& Zhu Hongjun (2011) proposed that larger the difference between FEPS and AEPS, more optimistic the analyst will be [1]. We learned the idea of Cao \& Zhu, and the practice of Wu Donghui (2005) [18], Jiang Guohua (2004)[19] and Hu Yiming (2005)[20], by using Formula (4), we calculated the forecast error of EPS (FEER), FEER is the difference between AEPS and FEPS. The formula is as follows:

$F E E R=\frac{F E P S-A E P S}{|A E P S|+0.5}=\frac{\varphi_{1}-\varphi_{2}}{\left|\varphi_{2}\right|+0.5}$

For the denominator cannot be zero, we added 0.5 to it.

Most scholars divided analysts into three groups, pessimistic, rational and optimistic [21], based on the optimism of analysts, and we followed this grouping idea in this paper. Scholars considered that if FEER is less than zero, the analyst is pessimistic tendency, otherwise, the analyst is optimistic tendency [19]. We chose 0.01 as the boundary to divide the optimistic group into two groups in detail: strong optimistic and weak optimistic. For the forecast error of weak optimistic group is small, we approximately treated them as rational. Thus, we divided analysts into three groups: pessimistic, rational and optimistic, and the grouping standard is as follows:

Table 1. Grouping Standard of Securities Analysts

\begin{tabular}{c|ccc}
\hline group & pessimistic & rational & optimistic \\
\hline standard & $F E E R \leq 0$ & $0<F E E R \leq 0.10$ & $F E E R>0.10$ \\
\hline
\end{tabular}

2.2.3. Fusion of evidence information in the group: After grouping, we need first fuse the evidence information in the same group. Considering both the simplicity of calculation and the importance difference of experts, we chose weighted average to fuse the evidence. The determination of the weight is based on the majority rule, big weight will be given if the similarity of evidences is high, otherwise, small weight will be given. The calculation of evidence distance is mainly based on Euclidean distance, Absolute distance and Chebyshev distance. CHEN[22] and Liu[23] have proved that Pignistic probability distance can be represented as evidence distance. Thus, we selected Pignistic probability distance to calculate the weight of evidence.

Give $\mathrm{Bel}_{1}$ and $\mathrm{Bel}_{2}$ as the basic probability assignment (BPA) functions under the framework $\Theta, B$ et $P_{m}$ and $B$ et $P_{m}$, are their corresponding Pignistic probability functions 
$[24,25]$. Calculate evidence weight $\omega_{j, i}$ by using Pignistic probability distance:

$$
\omega_{j, i}=\frac{\sum_{r=1}^{R_{j}} \operatorname{sim} B \operatorname{et} P_{E_{j, i}}^{E_{j, r}}}{\sum_{i=1}^{R_{j}} \sum_{r=1}^{R_{j}} \operatorname{sim} B \operatorname{et} P_{E_{j, i}}^{E_{j, r}}}
$$

Where, $\sum_{r=1}^{R_{j}} \operatorname{sim} B \operatorname{etP}_{E_{j, i}}^{E_{j, r}}$ is the sum of the Pignistic similarity between evidence $i$ in group $j$ and all the evidence in group $j, \sum_{i=1}^{R_{j}} \sum_{r=1}^{R_{j}} \operatorname{sim} \operatorname{Bet}_{E_{j, .}}^{E_{j, r}}$ is the sum of the Pignistic similarity among all the evidence in group $j$. The calculation of the similarity between evidence $E_{1}$ and evidence $E_{2}$ is as follows:

$$
\operatorname{simBetP} P_{E_{1}}^{E_{2}}=1-\operatorname{difBetP} P_{E_{1}}^{E_{2}}
$$

Where $\operatorname{difB}$ et $P_{E_{1}}^{E_{2}}$ is defined as:

$$
\left\{\begin{array}{l}
\operatorname{difBetP_{m_{1}}^{m_{2}}}=\max _{A \in \Theta}\left(\left|\operatorname{BetP}_{m_{1}}(A)-\operatorname{BetP}_{m_{2}}(A)\right|\right) \\
\operatorname{BetP}_{E}(\alpha)=\sum_{A \in \Omega, \alpha \in A} \frac{m(A)}{|A|}
\end{array}\right.
$$

When we fused all the evidence in a group into one piece of evidence, it is still a piece of evidence under a two-dimensional progressive framework. Suppose that there are $n$ pieces of evidence need to be fused, first, we divided them into 3 groups, then fused the evidence of each group into one piece of evidence, thus only 3 pieces of evidence need to be fused finally. This method can reduce the complexities of the fusion and increase the calculation efficiency, thus the convenience and usability of the model will be raised.

2.2.4. Correction and Fusion of Evidence among Groups: In the fusion of evidence among groups, we need to fit the characteristic of new evidence resource into the traditional evidence framework. The algorithm of Shafer in formula (2) provides the idea of fitting the second dimensional evidence into the first dimensional evidence.

The determination of correction factor $\alpha$ is a key problem when using formula (2). That is how to scientifically construct a mapping function $f:\left\{\varphi / \varphi_{1}, \varphi_{2}\right\} \rightarrow \alpha$, where the characteristic of evidence resource is mapped to the evidence correction factor $\alpha$. The information fusion model in this paper divided all the analysts into three groups: pessimistic, rational and optimistic, and fused the all the evidence in the same group into one piece of evidence, so the determination of $\alpha$ should follow two rules:

(1) Evidence correction factor must be an inverse relationship with FEER, high FEER means large correction degree, and $\alpha$ should be small. On the contrary, lower the FEER, bigger will the correction factor $\alpha$ be.

(2) For the new evidence of the three groups after fusion, the correction coefficient of rational group must be higher than the correction factor of pessimistic group and optimistic group. It means that the new evidence of pessimistic group and optimistic group must be given more modification.

The FEER of analysts in a group will be different, so the FEER of each group is obtained by the calculation of each analyst's FEER in the group: 
$F E E R_{G}=\frac{1}{k} \sum_{i}^{k} F E E R_{i}$

Considering about the two rules above, simplicity of calculation and comprehensibility of the results, we constructed Formula (9) to determine the correction factor $\alpha$ as follows:

$$
\left\{\begin{array}{l}
\alpha_{1}=\lambda_{1}-\left|F E E R_{1}\right| \\
\alpha_{2}=\lambda_{2}-\left|F E E R_{2}\right| \\
\alpha_{3}=\lambda_{3}-\left|F E E R_{3}\right| \\
1>\alpha_{1}>\alpha_{2}>0 \\
1>\alpha_{1}>\alpha_{3}>0
\end{array}\right.
$$

In the formula, $\alpha_{1}, \alpha_{2}, \alpha_{3}$ are the correction factors of rational group, optimistic group and pessimistic group. We chose eligible $\lambda_{1}, \lambda_{2}$ and $\lambda_{3}$ by traversal search or self-adaptive learning, and calculated the accuracy of the final fusion result of each $\lambda$. Finally, we used the $\lambda_{1}, \lambda_{2}$ and $\lambda_{3}$ under the highest result accuracy to calculate the correction factors of the groups.

After modifying the new evidence, we used Dempster's combination rule (Formula 3) to fusing all the evidence information.

By the fusion of evidence in the group and the fusion of evidence among groups, we finally turned the two-dimensional progressive framework to one dimensional progressive framework. This decreases the decision difficulty of decision maker and increases the decision efficiency.

2.2.5. Transform BPA to Probability: How to assign the probability of "uncertainty" information is a problem in transforming BPA to probability. In this paper, once a rating does not appear in the forecast results of analysts, the rating will not appear in final results. For example, if there is no analyst give "sell" to a stock, thus "sell" would not appear in the final fusion results. Besides, the discrimination of final results should also be considered in the transformation. Therefore, we chose the method, which the "uncertainty" information is prorated for the focal elements of fusion results, as Formula (10) shows.

$m *\left(\theta_{i}\right)=\frac{m\left(\theta_{i}\right)}{\sum_{\theta_{j} \in A} m\left(\theta_{j}\right)} m(A)+m\left(\theta_{i}\right)$

For results, which contain "uncertainty" information, only exist in the frame $\Theta$, thus the formula above can be simplified as:

$$
m *\left(\theta_{i}\right)=m\left(\theta_{i}\right)\left(\frac{m(\Theta)}{\sum m\left(\theta_{j}\right)}+1\right)
$$

In conclusion, the process of securities analysts' stock rating information fusion based on the evidential reasoning algorithm under two-dimensional progressive framework is as follows: 


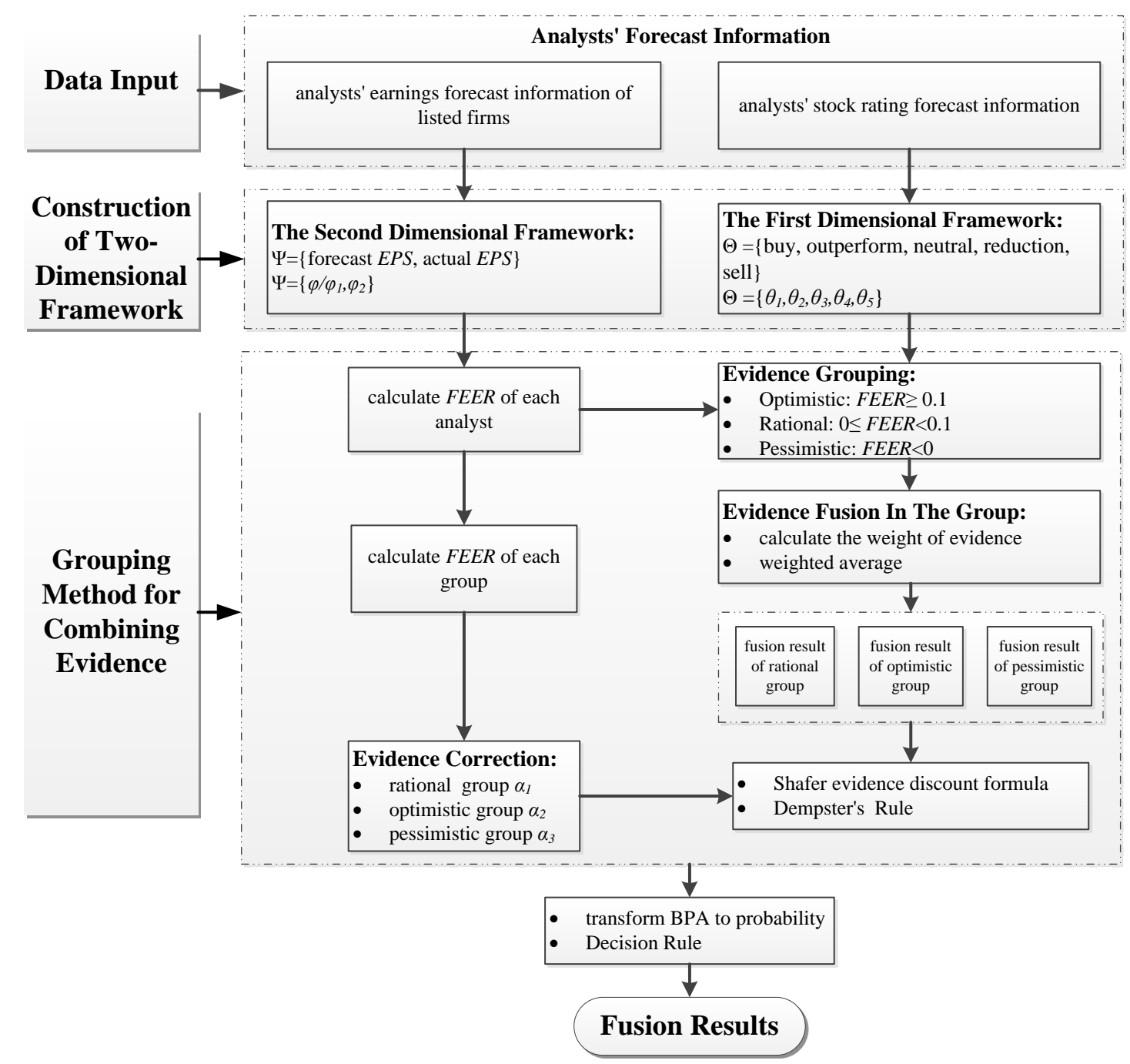

Figure 1. Process of Analysts' Stock Rating Information Fusion under Two-dimensional Progressive Framework

\section{Empirical Study}

\subsection{Data}

The grouping method for combining evidence under the two-dimensional progressive framework is suitable for cases with much evidence resource, thus we need to choose a large sample in this paper.

From the analysts' forecast information between January 1, 2008, and June 30, 2014, we selected stocks, which more than 10 analysts have given them stock rating in one day. And 3614 pieces of forecast information of stock rating have been selected in all. In the selected data, there are 1024 analysts and 236 stocks. Besides, in order to test the accuracy of the fusion results, we selected two indexes, return on investment (ROA) and index return of Hushen 300 Index. All the data are collected from Chinese GTA Database (CSMAR).

We compared ROA with Hushen 300 Index, and then calculated the abnormal return (AR). Event study is used to analyze forecast information of analysts, and the announcement time of forecast information is as event period. Supposed that we buy a stock at the date analysts' stock rating report published and hold it for 6 months (to 
simplify the calculation, we take it 120 trading days), and calculate AR of the stock. The calculation formula is as follow:

$A R=\prod_{t=1}^{120}\left(1+r_{t}\right)-\prod_{t=1}^{120}\left(1+R_{t}\right)$

Where $r_{t}$ is ROA in the $t$ day, $R_{t}$ is index return of Hushen 300 Index in the $t$ day.

The distribution of AR of the selected 236 stocks is shown as Figure 2.

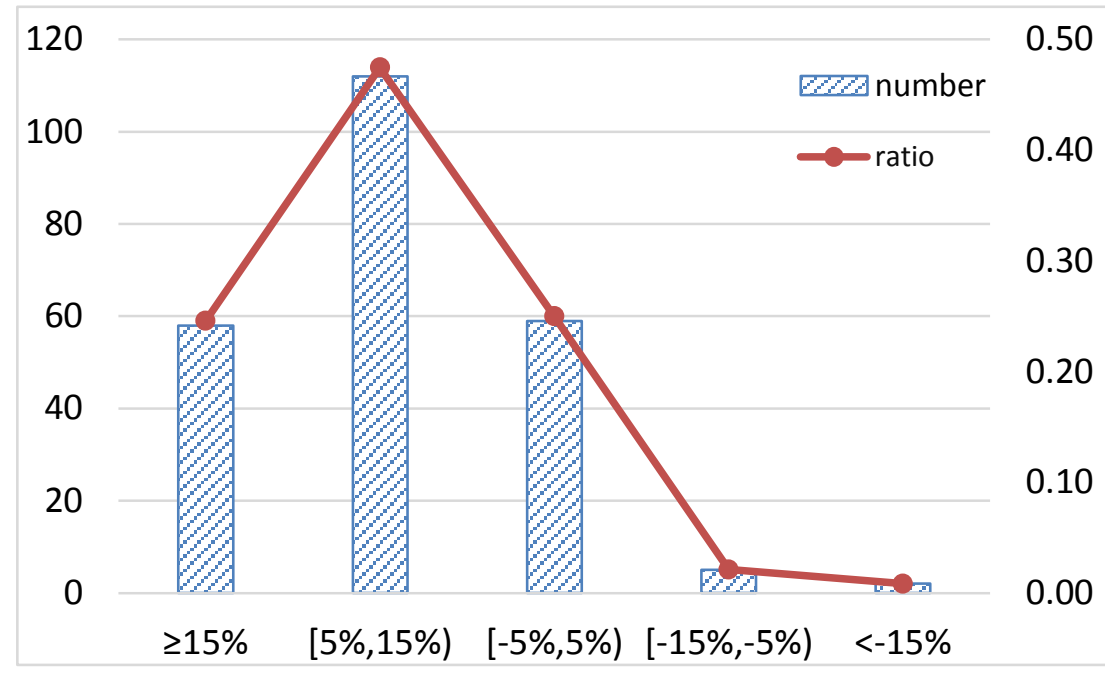

Figure 2. Distribution of AR

In order to verify the accuracy of the stock rating fusion results, we quantified the stock rating in this paper. Learn from the distinguish criterion of analysts' stock rating of HAITONG company and practice of Zhang Mei \& Wang Lei (2012) [21], the quantitative criteria of analysts' stock rating in this paper is as follows:

Table 2. Quantitative Criteria of Analysts' Stock Rating

\begin{tabular}{c|c}
\hline Stock Rating & Quantitative Criteria \\
\hline Buy & $\mathrm{AR} \geq 15 \%$ \\
Outperform & $5 \% \leq \mathrm{AR}<15 \%$ \\
Neutral & $-5 \% \leq \mathrm{AR}<5 \%$ \\
Reduction & $-15 \% \leq \mathrm{AR}<-5 \%$ \\
Sell & $\mathrm{AR}<-15 \%$ \\
\hline
\end{tabular}

\subsection{Application of the Information Fusion Model}

3.2.1. Grouping Analysts: To measure the optimistic tendency of the 1024 analysts accurately, we selected short-term (less than one year) earnings forecast information from 2002 to 2013, finally we obtained 64427 pieces of information. Suppose there are $n$ pieces of earnings forecast information of analyst $i$, then the FEER of him can be represented as the mean of the error of FEPS and AEPS. 
$F E E R_{i}=\overline{F E E R}=\frac{1}{n} \sum F E E R$

The statistical description of FEER of the 1024 analysts is shown as Table 3.

Table 3. Statistical Description of FEER

\begin{tabular}{cccccccc}
\hline Mean & Median & Mode & S.d & Variance & Min & Max & Samples \\
\hline 0.177 & 0.143 & 0 & 0.196 & 0.038 & -0.37 & 1.82 & 1024 \\
\hline
\end{tabular}

Based on the grouping rule in this paper, we divided the 1024 analysts into three groups:

Table 4. Grouping Criteria and Results

\begin{tabular}{c|ccc}
\hline Group Name & Grouping Criteria & Number of Experts & Mean of FEER \\
\hline Pessimistic & $F E E R \leq 0$ & 105 & -0.064 \\
Rational & $0<F E E R \leq 0.10$ & 332 & 0.070 \\
Optimistic & $F E E R>0.10$ & 587 & 0.281 \\
\hline
\end{tabular}

3.2.2. Evidence fusion in the group: We chose one specific stock from the 236 stocks as an example to show the fusion process. The related data of this stock is as follows:

Table 5. Related Data of the Stock

\begin{tabular}{c|ccccccccc}
\hline Stock & & & & & & & & & \\
Rating & $\mathrm{A}$ & $\mathrm{B}$ & $\mathrm{C}$ & $\mathrm{A}$ & $\mathrm{C}$ & $\mathrm{B}$ & $\mathrm{A}$ & $\mathrm{A}$ & $\mathrm{B}$ \\
Tendency & $\mathrm{R}$ & $\mathrm{R}$ & $\mathrm{R}$ & $\mathrm{R}$ & $\mathrm{R}$ & $\mathrm{P}$ & $\mathrm{P}$ & $\mathrm{P}$ & $\mathrm{P}$ \\
FEER & 0.033 & 0.030 & 0.031 & 0.032 & 0.030 & -0.032 & -0.032 & -0.047 & -0.048 \\
\hline Stock & & & & & & & & & \\
Rating & $\mathrm{B}$ & $\mathrm{A}$ & $\mathrm{C}$ & $\mathrm{A}$ & $\mathrm{B}$ & $\mathrm{B}$ & $\mathrm{B}$ & $\mathrm{B}$ & \\
Tendency & $\mathrm{P}$ & $\mathrm{O}$ & $\mathrm{O}$ & $\mathrm{O}$ & $\mathrm{O}$ & $\mathrm{O}$ & $\mathrm{O}$ & $\mathrm{O}$ & \\
FEER & -0.048 & 0.172 & 0.143 & 0.292 & 0.157 & 0.127 & 0.126 & 0.154 &
\end{tabular}

NOTE: (1)Stock rating A, B, C, D, E represent buy, outperform, neutral, reduction and sell, similarly hereinafter. (2)Tendency R, P, O represent rational, pessimistic and optimistic.

First, we should transform the information of the stock rating and tendency of analysts into BPA function. For there is no "uncertainty" in the stock rating information of analysts, we can transform the information in Table 5 to BPA by the following mapping: A can be represented as $\{1,0,0,0,0\}, \mathrm{B}$ can be represented as $\{0,1,0,0,0\}, \mathrm{C}$ can be represented as $\{0,0,1,0,0\}$, D can be represented as $\{0,0,0,1,0\}$ and $\mathrm{E}$ can be represented as $\{0,0,0,0,1\}$.

Second, calculate the weight of the 5 evidence in rational group. Calculate the Pignistic evidence distance between the first piece of evidence and all the evidence based on Formula (6) and (7), and $\operatorname{difB}$ etP is $(0,1,1,0,1)$, and the corresponding Pignistic evidence similarity $\operatorname{sim} B$ et $P$ is $(1,0,0,1,0)$. Similarly, we can calculate Pignistic evidence similarity between all the evidence. By using Formula (5), we can obtain the weight of each evidence, they are $(0.222,0.112,0.222,0.222,0.222)$. By using weight average method as Formula (1), we can calculate the fusion results of the 5 evidence: 
$m(A)=0.444 ; m(B)=0.112 ; m(C)=0.444 ; m(D)=0 ; m(E)=0$.

In the same way, the BPA can be calculated in pessimistic group and optimistic group. The results are shown in Table 6 .

Table 6. Evidence Fusion Results in the Same Group

\begin{tabular}{|c|c|c|c|c|c|}
\hline $\begin{array}{l}\text { Stock rating } \\
\text { Tendency }\end{array}$ & A & B & $\mathrm{C}$ & $\mathrm{D}$ & $\mathrm{E}$ \\
\hline Pessimistic & 0.308 & 0.692 & 0.000 & 0.000 & 0.000 \\
\hline Optimistic & 0.190 & 0.762 & 0.048 & 0.000 & 0.000 \\
\hline Rational & 0.444 & 0.112 & 0.444 & 0.000 & 0.000 \\
\hline
\end{tabular}

3.2.3. Correction and fusion of evidence among groups: By using Formula (13), we can calculate the FEER of experts in each group of the example above: FEER of the rational group is $0.031\left(F E E R_{l}\right)$, the $F E E R$ of the optimistic group is $0.167\left(F E E R_{2}\right)$ and FEER of the pessimistic group is $-0.041\left(\right.$ FEER $\left._{3}\right)$.

To obtain the optimal evidence correction factor, we use traversing method to determine the $\lambda$ in Formula (9). In the 236 stocks, the maximum FEER of the rational group is 0.098 , the maximum FEER of the optimistic group is 0.470 and the minimum FEER of the pessimistic group is -0.256 . For that the correction factor $\alpha$ must be positive, the initial value of $\lambda_{1}, \lambda_{2}, \lambda_{3}$ is set as $(0.01,0.50,0.30)$, and the maximum value is set as $(1.00,1.40,1.20)$. Set the step length of $\lambda_{1}, \lambda_{2}, \lambda_{3}$ all be 0.1 , and then try to get the optimal value after searching, after obtaining the optimal value, change the step length to 0.05 , and try again to get the optimal value after searching. The calculations show that when $\lambda_{I}$ $=0.80, \lambda_{2}=0.50$, and $\lambda_{3}=0.50$, the accuracy of the output results is the highest. Therefore, the evidence correction factor $\alpha$ is calculated as follow:

$\left\{\begin{array}{l}\alpha_{1}=0.80-\left|F E E R_{1}\right| \\ \alpha_{2}=0.50-\left|F E E R_{2}\right| \\ \alpha_{3}=0.50-\left|F E E R_{3}\right|\end{array}\right.$

After correcting the new evidence in the three groups, we use Dempster Rule to fuse all the evidence information, and then we use Formula (11) to transform BPA into probability. The fusion results and transformation results of the example above are as follows:

Table 7. Fusion Results and Transformation Results of Evidence among Groups

\begin{tabular}{c|cccccc|c}
\hline Evidence framework & $\mathrm{A}$ & $\mathrm{B}$ & $\mathrm{C}$ & $\mathrm{D}$ & $\mathrm{E}$ & $\Theta$ & $\begin{array}{c}\text { Correction } \\
\text { factor } \alpha\end{array}$ \\
\hline Pessimistic group & 0.308 & 0.692 & 0.000 & 0.000 & 0.000 & 0.00 & 0.459 \\
Optimistic group & 0.190 & 0.762 & 0.048 & 0.000 & 0.000 & 0.00 & 0.333 \\
Rational group & 0.444 & 0.112 & 0.444 & 0.000 & 0.000 & 0.00 & 0.769 \\
\hline Fusion results & 0.126 & 0.684 & 0.046 & 0.000 & 0.000 & 0.145 & ---- \\
\hline Transformation results & 0.147 & 0.799 & 0.054 & 0.000 & 0.000 & 0.000 & ---- \\
\hline
\end{tabular}




\subsection{Analysis of the Fusion Results}

After the fusion, we need a selection rule to determine the final fusion results of analysts' stock rating information. In general, maximum rule $\left(\operatorname{Max}\left[P\left(\theta_{i}\right)\right]\right)$ is selected to make decision, that means choosing focal element with the maximum probability in the framework as the final result.

In order to compare our model to traditional model, we first chose the maximum rule to obtain the final fusion results. Analyze all the 236 cases, we found that, under traditional model there are 89 right cases in the fusion results (the accuracy is $37.71 \%$ ), and there are 8 unrecognized simples (several focal elements have the same probability), and the results under the two-dimensional framework in this paper shows that: there are 137 right cases in the fusion results, the accuracy is $58.05 \%$, obviously superior to the accuracy of traditional model.

In practice, the probability of a focal element may be the maximum one among all the propositions, but the probability value itself may be small. For further comparison of our model and traditional model, we added one more selection rule based on the maximum rule:

$\operatorname{Max}\left[P\left(\theta_{i}\right)\right]>\partial$

$\partial$ is the minimal and acceptable value of the maximum probability. Given $\partial$, when the maximum probability of a focal element in the framework is larger than $\partial$, then output result is considered to be right. If the maximum probability of a focal element in the framework is less than $\partial$, then it is considered to be that the result is unable to judge or it is wrong. While, the determination of $\partial$ is based on subjective preference and values of the decision maker, when $\partial=0$, the decision rule is the maximum rule. Table 8 has shown the change of accuracy of the results as $\partial$ varies.

Table 8. Comparison of the Fusion Results of Model as $\partial$ varies

\begin{tabular}{c|cc|cc}
\hline \multirow{2}{*}{$\partial$} & \multicolumn{2}{|c|}{ Our model } & \multicolumn{2}{c}{ Traditional statistic model } \\
\cline { 2 - 5 } & Right cases & Accuracy & Right cases & Accuracy \\
\hline 0.3 & 137 & $58.05 \%$ & 89 & $37.71 \%$ \\
0.4 & 136 & $57.63 \%$ & 89 & $37.71 \%$ \\
0.5 & 130 & $55.08 \%$ & 76 & $32.20 \%$ \\
0.6 & 98 & $41.53 \%$ & 46 & $19.49 \%$ \\
0.7 & 70 & $29.66 \%$ & 10 & $4.24 \%$ \\
0.8 & 52 & $22.03 \%$ & 2 & $0.85 \%$ \\
0.9 & 24 & $10.17 \%$ & 0 & $0.00 \%$ \\
1 & 0 & $0.00 \%$ & 0 & $0.00 \%$ \\
\hline
\end{tabular}

Based on the maximum rule, there are 137 right cases of the fusion results under the two-dimensional progressive framework, and there are only 89 right cases under traditional model. Further analysis will be focus on the certainty degree (the distribution of the maximum probability) of the fusion results of our model and traditional statistic model. The comparison is as Figure 3: 


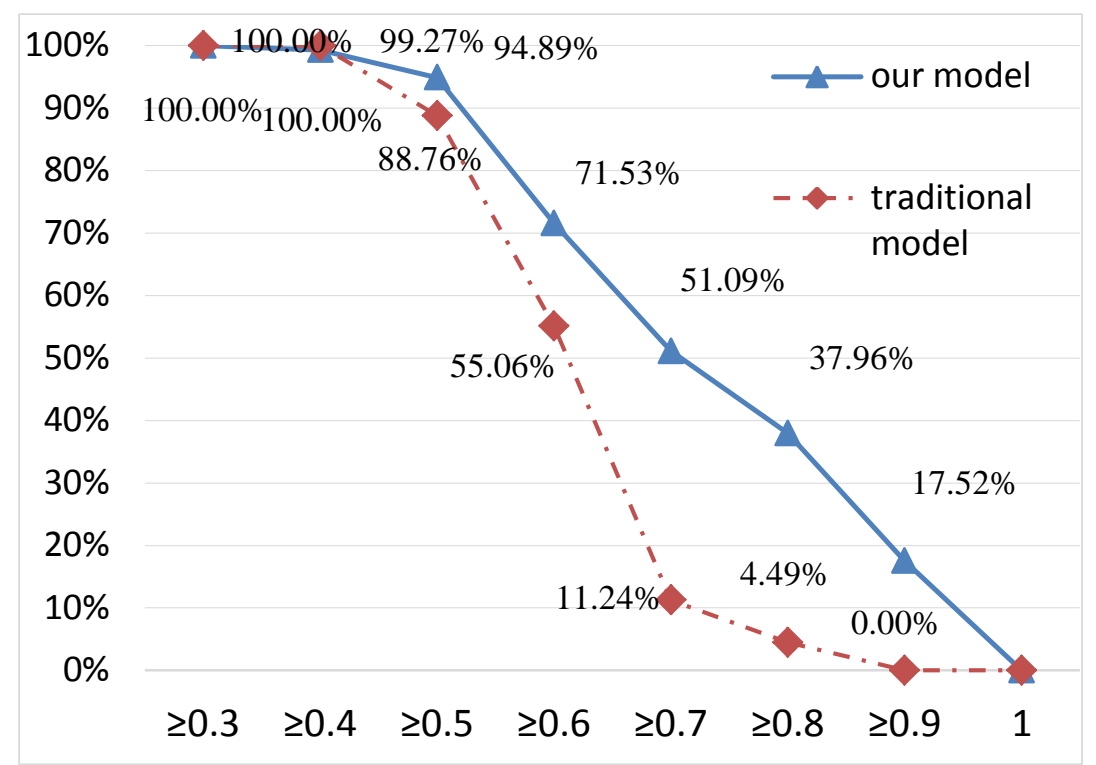

Figure 3. Comparison of Certainty Degree of the Two Models

It is obvious that applying the two-dimensional progressive framework to the fusion of analysts' forecast information of stock rating, the certainty of the fusion results have been improved. Given a stock as an example, the fusion results is "sell" under both our model and traditional model, while $66.67 \%$ analysts of traditional model will give "sell", while our model is $87.90 \%$. Furthermore, for the 85 right cases in both two models, the certainty of $95.29 \%$ of them has been raised.

The following analysis is on the Discrimination of the two models. The discrimination is the difference between the maximum probability and the second maximum probability of the focal elements in the framework. Larger the discrimination, the fusion results will be more useful to decision makers.

$D c m=\operatorname{Max}\left[P\left(\theta_{i}\right)\right]-\operatorname{SecMax}\left[P\left(\theta_{j}\right)\right]$

Where $\operatorname{Sec} \operatorname{Max}\left[P\left(\theta_{j}\right)\right]$ is the second maximum probability of the focal elements.

The distribution of Discrimination of the two models is shown as Figure 4. The Discrimination declining of our model is slower than traditional statistic model. For the 85 right cases in both two models, the Discrimination of 71 of them $(83.53 \%)$ has been raised. So the model in this paper can raise the Discrimination of the fusion results. 


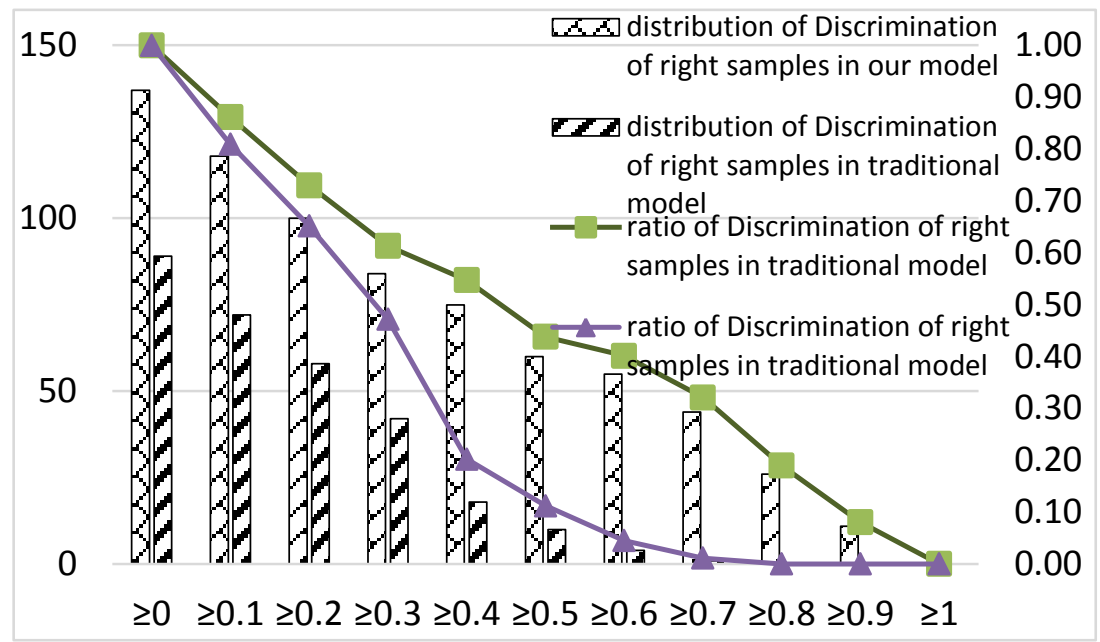

Figure 4. Comparison of Discrimination of the Two Models

In conclusion, the fusion model for securities analysts' stock rating information based on the evidential reasoning algorithm under two-dimensional progressive framework can improve the accuracy, certainty and discrimination of the fusion results, and it is more convenient for decision makers to confirm the final fusion results.

\section{Conclusion}

Analysts' forecast reports contain large amount of information, which can effectively reduce the degree of information asymmetry in securities markets. Collecting and fusing forecast information of different analysts can effectively reduce the difficulty in using analysts' forecast reports for individual investors, thus can improve the utilization of analysts' forecast reports. In this paper, evidential reasoning algorithm under two-dimensional progressive framework was applied to the fusion of securities analysts' stock rating information. The analysis on the output results has shown that: the accuracy, certainty and the discrimination of the fusion results have been improved.

\section{Acknowledgements}

This work is partially supported by National Natural Science Foundation of China, Grant Nos. 71071048, 71302063; the Soft Science Research Plan in Anhui Province, Grant No. 1402052006. We gratefully appreciate these supports.

\section{References}

[1] C. Sheng and Z. Hongjun, "Every potter praises hit pot: brokerage' proprietary trading and analysts' optimism", J. Management World.7: 005 (2011).

[2] Z. Hongjun, H. Xianjie and T. Lin, "Can Chinese analysts improve the efficiency of capital market ?Based on stock empirical envidence of price synchronicity and stock price informativeness", J. Journal of Financial Research. (02A): (2007), pp. 110-121.

[3] G. Jie and H. Jieying, "A Study on the Effectiveness of Chinese Security Analysts' Earnings Forecasting Behavior", J. Economic Research Journal.11: (2009), pp. 55-67.

[4] Brown N. C. and Christensen T. E., "The Quality of Street Cash Flow from Operations", J. Review of Accounting Studies, vol. 19, no. 2, (2014), pp. 913-954.

[5] W. Yuxi, H. Jianqiao and X. Jun, "Do Star Analysts' Recommendations from Top Brokers have more Investment Value?An Empirical Study Based on Reputation”, J. Journal of Industrial Engineering and Engineering Management, vol. 26, no. 3, (2012).

[6] Y. Yihua and Z. Yunjun, "Study of Influencing Factors on Analysts Forecast Accuracy: Based on Panel Data of Shenzhen Stock Exchange”, J. Accounting and Finance, no. 2, (2014), pp. 91-95. 
[7] W. Chaopeng, Z. Fangbiao and Y. Shijie, "Are Analysts Earnings Forecasts and Investment Recommendations Objective?”, J. China Economic Quarterly, no. 3, (2013), pp. 935-958.

[8] Y. Lushi, H. Fang, W. Lina and L. Xiaojie, "Research on Security Analyst Follow-up Behavior Under Conflicts of Interest- An Exemplified Study Based on Panel Data", J. Chinese Journal of Management Science, vol. 6, (2014).

[9] Z. Wweidong, "Research on Theory of Evidence Based and Internet Oriented Intelligent Decision Support Systems", D. HeFei University of Technology, (2003)

[10] Kubik J. D. S. A. H. H., "Security Analysts' Career Concerns and Herding of Earnings Forecasts", J. RAND Journal of Economics, no. 1, (1998), pp. 121-144.

[11] Shafer G., "A mathematical theory of evidence", Princeton: Princeton university press, (1976).

[12] Frikha A. and Moalla H., "Analytic hierarchy process for multi-sensor data fusion based on belief function theory", J. European Journal of Operational Research, (2014).

[13] H. Jinhai, Y. Zhiguo and D. Xusheng, "Research of Decision Fusion Diagnosis of Aero-engine Rotor Fault Based on Improved D-S Theory", J. Acta Aeronautica et Astronautica Sinica, no. 2, (2014), pp. 436-443.

[14] Deng X., Zheng X. and Su X., "An evidential game theory framework in multi-criteria decision making process", J. Applied Mathematics and Computation, (2014), pp. 244:783-793.

[15] Y. Shanlin, Z. Wweidong and R. Minglun, "Learning based combination of expert opinions in securities market forecasting", J. Journal of Systems Engineering, vol. 19, no. 1, (2004), pp. 94-98.

[16] Z. Hongtao, Z. Weidong, W. Hui and W. Yong, "An evaluation method for scientific research project selection based on the evidential reasoning algorithm under multi-dimensional frames of discernment", J. Science Research Management, vol. 6, (2013), pp. 122-128.

[17] Z. Weidong, S. Yibo and W. Yong, "Research on group method for combining based on the characteristic of evidence resource in two-dimensional framework", The 18th academic annual conference of the Systems Engineering Society of China. (2014) HeFei, China.

[18] W. Donghui and X. Zuyun, "The Investment Value of Financial Analysts' Earnings Forecasts: Evidence from the A-Share Markets in Shanghai and Shenzhen", J. Accounting Research, vol. 8, (2005).

[19] J. Guohua, "Empirical Study on Analysts' Accounting Earrings Forecast of Chinese Listed Firms", J. Economic Science, vol. 6, (2004).

[20] H. Yiming and L. Wenxiong, "Information Comprehension, Analysis Ability and Analysis QualityInvestigation and Analysis on Chinese Analysts", J. Journal of Financial Research, no. 2, (2005), pp. 46-58.

[21] Z. Mei, W. Lei and W. Lina, "Research on Securities Analysts Recommendation's Investment Value and Accuracy", J. Journal of HeFei University of Technology (Social Science Edition), no. 3, (2012), pp. 7-11.

[22] C. Shengqun and W. Yingming, "Optimal Combination of Evidence Based on Pignistic Probability Distance", J. Information and Control, no. 2, (2013), pp. 213-217.

[23] Liu W., "Analyzing the degree of conflict among belief functions", J. Artificial Intelligence, vol. 170, no. 11, (2006), pp. 909-924.

[24] Aregui A. and Denoeux T., "Constructing consonant belief functions from sample data using confidence sets of pignistic probabilities", J. International Journal of Approximate Reasoning, vol. 49, (2008), pp. 575-594.

[25] Smets P., "Decision making in the TBM: the necessity of the pignistic transformation", J. International Journal of Approximate Reasoning, vol. 38, no. 2, (2005), pp. 133-147.

\section{Authors}

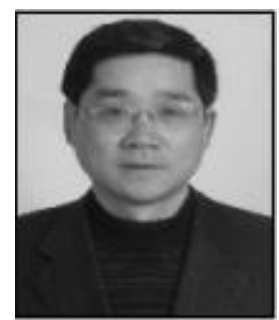

Weidong Zhu, is a professor of Hefei University of Technology, and his research area concludes: Decision Science, Decision Support System, Accounting information and decision-making. 


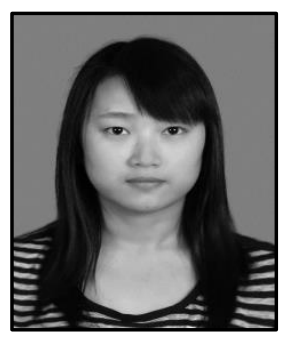

Yiling Wang, is a doctoral candidate of Hefei University of Technology, and her research area concludes: Decision Science, Accounting information and decision-making.

(Corresponding author: wangyiling@mail.hfut.edu.cn)

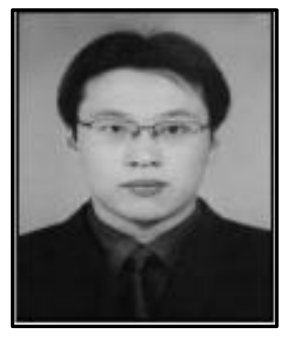

Yong Wu, is an associate professor of Hefei University of Technology, and his research area concludes: Decision Science, Knowledge Sharing.

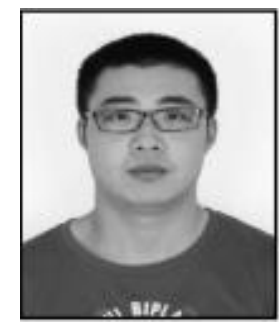

Yibo Sun, is a Doctor of Hefei University of Technology, and his research area concludes: Decision-making theory and method 
International Journal of Security and Its Applications

Vol. 10, No. 7 (2016) 